\title{
ENFERMERÍA. PRESCRIPCIÓN DE FÁRMACOS Y PRODUCTOS SANITARIOS.
}

Antonia Martín Perdiz.

Profesora Colaboradora. Departamento de Enfermería y Fisioterapia. Universitat de les Illes Balears.

La Ley 28/2009, de 30 de diciembre, que modifica la Ley 29/2006, de 26 de julio, de garantías y uso racional de los medicamentos y productos sanitarios, ha hecho posible lo que se conoce comúnmente como "prescripción enfermera". Antes de pasar a profundizar en la prescripción de fármacos y productos sanitarios, es preciso clarificar que la Prescripción Enfermera es un concepto mucho más amplio. Utilizando la definición de Bulecheck y McCloskey (1992), podríamos decir que es "cualquier cuidado directo que la enfermera realiza en beneficio del cliente"[1]. Este cuidado directo incluye:

- Los tratamientos iniciados por la enfermera.

- Los tratamientos iniciados en función del diagnóstico médico.

- La realización de actividades diarias esenciales para el cliente.

La adecuada prescripción de fármacos y productos sanitarios vinculados a los cuidados enfermeros es una competencia imprescindible para un ejercicio profesional responsable y autónomo y parece razonable pensar que el manejo de fármacos debería incluirse en el Libro Verde de la Directiva Europea sobre Competencias Profesionales. Ésta es la opinión del $91.6 \%$ de los participantes en la encuesta elaborada este año por la European Federation of Nurse 
Educators, que pretende usar los datos obtenidos para actualizar la Directiva Europea 2005/36/EC relativa al reconocimiento de cualificaciones profesionales.

La mencionada Ley 28/2009 fue aprobada tras varios años de reivindicaciones y se sustenta sobre cuatro pilares: la Ley de Ordenación de las Profesiones Sanitarias, la sinergia entre diferentes colectivos enfermeros, el aumento del nivel de la formación de Grado y Postgrado en Enfermería, y, por último, el reconocimiento a una tarea de prescripción que las enfermeras llevaban a cabo hasta ese momento al margen de la legalidad vigente.

La Ley 44/2003, de 21 de noviembre, de Ordenación de las Profesiones Sanitarias reconoce que la Enfermería es una profesión independiente y regula nuestro ámbito competencial. Concretamente, el artículo 7.2.a establece que corresponde a las enfermeras «la dirección, evaluación y prestación de los cuidados de Enfermería orientados a la promoción, el mantenimiento y recuperación de la salud, así como a la prevención de enfermedades y discapacidades».

Las reivindicaciones a favor de la regulación de la prescripción enfermera aunaron al colectivo enfermero y encontraron apoyo en diferentes gobiernos autonómicos, especialmente de la Junta de Andalucía y la Generalitat de Catalunya. Cabe citar aquí que la Junta de Andalucía fue pionera al promulgar el Decreto 307/2009 que regula la actuación de las enfermeras en el ámbito de la prestación farmacéutica del Sistema Sanitario Público de Andalucía.

Por lo que respecta al ámbito enfermero, las Universidades, Sociedades científicas como SCELE, y la Organización colegial enfermera española, elaboraron sendos estudios y documentos de trabajo que sirvieron para sustentar la necesidad de este tipo de prescripción. Cabe destacar también aquí por su especial relevancia el Informe del Consell de Col/legis de Diplomats en Infermeria de Catalunya del 2007 que incluyó un extenso catálogo susceptible de ser prescrito por enfermeras, o el Listado de medicamentos y productos sanitarios prescritos por las enfermeras en España elaborado también el 2007 por el Consejo General de Enfermería.

El aumento de la formación enfermera en nuestro país ha conllevado que queramos asumir nuevas competencias profesionales. El Real Decreto 450/2005, de 22 de abril, sobre especialidades de Enfermería, la puesta en marcha de los estudios oficiales de Máster y Doctorado y, recientemente, el inicio de los estudios de Grado en Enfermería 
configuran un marco formativo que sitúa a las enfermeras españolas en un punto de partida excelente para ser competitivas en el ámbito laboral. Adicionalmente, la libre circulación de profesionales que posibilita la Unión Europea ha estimulado el que las enfermeras españolas quieran asumir nuevas competencias que nos sitúen en igualdad de condiciones respecto al resto de enfermeras europeas y del ámbito anglosajón. Siete son los países que cuentan con legislación que permite la prescripción enfermera de fármacos y productos sanitarios vinculados a los cuidados: Australia, Canadá, Irlanda, Nueva Zelanda, Suecia, Inglaterra y los Estados Unidos (Kroezen et al., 2011). Este tipo de prescripción funciona con toda normalidad en Inglaterra desde el año 2000, país que cuenta con más de 10.000 enfermeras acreditadas para prescriptoras independientes.

Cabe recordar aquí que la Ley 29/2006 dejó la prescripción enfermera al margen de la legalidad. La Ley 28/2009 vino pues a reconocer una actividad necesaria en el sistema sanitario y que se estaba llevando a cabo de manera más o menos habitual. El estudio elaborado el 2007 por el Consejo General de Enfermería y que recogió más de 1700 aportaciones de enfermeras asistenciales evidenció que la prescripción de fármacos y productos sanitarios era habitual, siendo los productos más prescritos los analgésicos, hidrocoloides, antiinflamatorios y antibióticos.

En el contexto de crisis económica actual, y con el recorte de servicios sanitarios que se está dando en todo nuestro país, es adecuado revisar las implicaciones que la modificación de la Ley 29/2006 supone para nuestra profesión. En la mayoría de países existen dos tipos de prescripción enfermera:

- Autónoma: está regulada legalmente y es ejecutada por enfermeras que están específicamente cualificadas para ello.

- Colaborativa: la enfermera prescribe sustancias contenidas en un catálogo y debe consultar con el médico u odontólogo antes de llevar a cabo la prescripción concreta, aunque la supervisión directa de éste no es imprescindible).

La Ley 28/2009 recoge ambos tipos de prescripción en el artículo 77, apartado 1:

(...) los enfermeros, de forma autónoma, podrán indicar, usar y autorizar la dispensación de todos aquellos medicamentos no sujetos a prescripción médica y los productos sanitarios, mediante la correspondiente orden de dispensación.

El Gobierno regulará la indicación, uso y autorización de dispensación de 
determinados medicamentos sujetos a prescripción médica por los enfermeros, en el marco de los principios de la atención integral de salud y para la continuidad asistencial, mediante la aplicación de protocolos y guías de práctica clínica y asistencial, de elaboración conjunta, acordados con las organizaciones colegiales de médicos y enfermeros y validados por la Agencia de Calidad del Sistema Nacional de Salud.

El Ministerio de Sanidad y Política Social con la participación de las organizaciones colegiales, referidas anteriormente, acreditará con efectos en todo el Estado, a los enfermeros para las actuaciones previstas en este artículo.

Nuestro colectivo considera que estamos capacitadas para prescribir fármacos y productos sanitarios (Cabrera-Jaime et al, 2011). Así pues, la redacción del artículo 77 suscitó en su momento una gran polémica en la profesión dado que ningún otro de los profesionales recogidos en la ley como prescriptores, esto es, médicos, odontólogos y podólogos, precisan obtener acreditación alguna tras obtener su título universitario. Esta discriminación fue objeto de protestas por parte del colectivo pero, dada la necesidad de agilizar la puesta en marcha de la prescripción enfermera, la Organización Colegial y las Universidades se pusieron manos a la obra para conseguir que las enfermeras podamos obtener la acreditación.

Así pues, y en respuesta a la Ley 28/2009, la Organización Colegial ha venido organizando un curso específico en la práctica totalidad de las Comunidades Autónomas. A modo de ejemplo de la demanda generada, podemos citar al Colegio Oficial de Enfermería de Barcelona, cuyo curso fue seguido por 873 enfermeras en el 2010, o el Colegio Oficial de Enfermería de Tenerife, que ha llegado algo más de 900 enfermeras.

En cuanto a la respuesta de las Universidades, éstas han puesto en marcha estrategias diversas. Desde la de la Universidad Complutense de Madrid que ha incluido en el plan de estudios del Grado en Enfermería una asignatura concreta que ha denominado Prescripción, hasta otras universidades que han explicitado descriptores vinculados a la prescripción en los programas de diversas asignaturas.

En cuanto a los retos que supondrá la puesta en marcha efectiva de la prescripción enfermera en España, podemos citar los vinculados al aumento de responsabilidad legal de las enfermeras prescriptoras. Esta situación se ha vivido ya en otros países y vale la pena poner los medios para evitar caer en los mismos errores que nos advierten quienes ya han implementado la 
prescripción enfermera. La literatura recoge dificultades como los problemas derivados de las dificultades en el acceso a los programas informáticos de emisión de recetas, o la falta de formación continuada (Courtenay et al, 2007). Así mismo, cabe destacar que la prescripción debe ir vinculada ineludiblemente a la evidencia disponible y en nuestro país el uso cotidiano de la evidencia es todavía una asignatura pendiente (Gea-Sánchez, 2010). Por otra parte, los trámites burocráticos asociados a la prescripción pueden suponer un aumento de nuestra carga de trabajo (Drennan et al., 2009) en un momento de recortes de personal.

Por último, cabe destacar la gran oportunidad que la prescripción nos brinda para hacer más relevante nuestra aportación a la sociedad y afianzar nuestro rol en las instituciones sanitarias, especialmente en un momento delicado para las direcciones de Enfermería en los hospitales y en Atención Primaria (Artigas, 2011; Guzmán Fernández, 2011). La prescripción enfermera es necesaria para la plena autonomía enfermera en nuestro país (Romero, 2011). En países como Irlanda la puesta en marcha de la prescripción enfermera ha sido positiva para el desarrollo profesional de las enfermeras y matronas. Ha elevado su autonomía, su satisfacción laboral, y les ha permitido utilizar con más plenitud sus habilidades profesionales, con la consiguiente mejora de los cuidados ofrecidos a los pacientes. Éstos por su parte se han mostrado muy favorables a la prescripción enfermera puesto que reduce los tiempos de espera y les facilita el acceso al tratamiento que precisan (Drennan et al., 2009; While \& Biggs, 2004).

En tiempos de crisis como los que vivimos, la prescripción de fármacos y productos sanitarios vinculados a los cuidados puede ayudar a que el sistema sanitario funcione de manera más eficiente. Es beneficiosa para nuestra profesión y para los usuarios de nuestros servicios. Así pues, la implantación de la prescripción enfermera es un reto que vale la pena afrontar con ilusión.

\section{Documentos referenciados:}

Artigas, M. (2011). Pérdida de poder de las direcciones de enfermería de los hospitales. Enfermería clínica, 21(3), 127-8.

Cabrera-Jaime, S., Estrada-Masllorens, J. M., \& Fernández-Ortega, P. (2011). Conocimiento y satisfacción de la aprobación de la prescripción enfermera (Ley 28/2009) en enfermeras de formación máster y especialidad de la Universidad de Barcelona. Enfermería clínica, 21(2), 84-90. 
Courtenay, M., Carey, N., \& Burke, J. (2007). Independent extended and supplementary nurse prescribing practice in the UK: a national questionnaire survey. International journal of nursing studies, 44(7), 1093101.

Drennan, J., Naughton, C., Allen, D., Hyde, A., Felle, P., O'Boyle, K., Pearl, T., et al. (2009). National Independent Evaluation of the Nurse and Midwife Prescribing Initiative. Evaluation. Dublin: Health Service Executive.

Gea-Sánchez, M. (2010). Conocimiento, actitudes y barreras percibidas por las enfermeras para integrar evidencia científica en la práctica clínica en un hospital universitario. Enfermería clínica, 20(5), 313-4.

Guzmán Fernández, M. Á. (2011). Pérdida de poder de las direcciones de enfermería de Atención Primaria. Enfermería clínica, 21(4), 187-8.

Kroezen, M., van Dijk, L., Groenewegen, P. P., \& Francke, A. L. (2011). Nurse prescribing of medicines in Western European and Anglo-Saxon countries: a systematic review of the literature. BMC Health Services Research, 11(1), 127. BioMed Central Ltd.

Romero, Á. (2011). Prescripción enfermera en España. Se hace camino al andar. Revisión de la literatura. Rol de Enfermería, 34(6), 12-23.

[1] Consejo General de Enfermería. Marco referencial para la prescripción enfermera. Documento Base elaborado por el Consejo General de Enfermería y presentado a los medios de comunicación el 23 de Marzo de 2006. 